\title{
Dinámica epidemiológica del dengue en Isla de Pascua
}

\author{
Mauricio Canals, Christian González, Andrea Canals y Daniela Figueroa
}

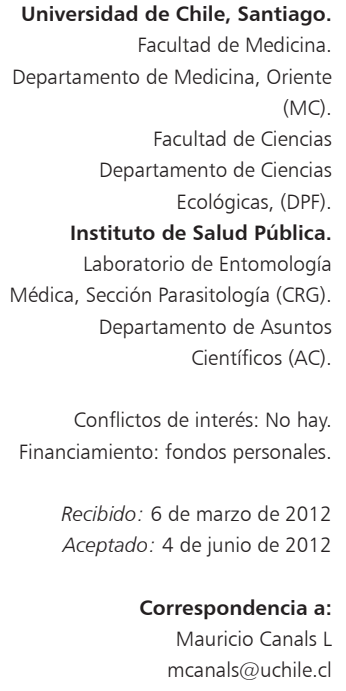

\section{Epidemiological dynamics of dengue on Easter Island}

Dengue is considered an emerging disease with an increasing prevalence especially in South America. In 2002, an epidemic of classic Dengue (DENV-1) occurred unexpectedly on Easter Island, where it had never been detected before. It reappeared in 2006-2007 and 2008, 2009 and 2011. The aim of this study was to estimate the most relevant parameters of the epidemiological dynamics of transmission of Dengue on Easter Island and to model the dynamics since 2002, comparing the predictions with the actual situation observed. Of the total cases, $52.27 \%$ were females and $47.73 \%$ men. The average age of infection was $31.38 \pm 18.37$ years, similar in men and women. We estimated the reproductive number $\mathrm{R}_{0}=3.005$ with an $\mathrm{IC}_{0,95}[1.92,4.61]$. The inter-epidemic period reached an estimated $\mathrm{T}=5.20$ to 6.8 years. The case simulation showed recurrent epidemics with decreasing magnitude (damped oscillations), which is a known phenomenon in models of dengue and malaria. There was good qualitative fit to the epidemiological dynamics from 2002 onwards. It accurately predicted the rise in cases between 2006 and 2011. The predicted number of cases during the 2002 epidemic is greater than the confirmed cases and the predicted epidemic was faster than notified cases. Interepidemic period in the simulation was 6.72 years between 2002 and 2008 and 4.68 years between 2008 and 2013. From the theoretical perspective, the first epidemic had affected $94 \%$ of the population (approximately 3500 cases), but 639 were reported suggesting underreporting and a lot of sub-clinical cases occurred. Future epidemic of decreasing size are expected, although the main danger are epidemics of hemorrhagic dengue fever resulting from the introduction of different dengue virus serotypes.

Key words: Dengue, Easter Island, Models, Epidemics.

Palabras clave: Dengue, Isla de Pascua, modelos, epidemia.

\section{Introducción}

$\mathrm{E}$ 1 dengue es una enfermedad producida por un arbovirus de la Familia Flaviviridae, transmitido por la picadura de algunas especies de mosquitos de los géneros Aedes (o Stegomyia) como A. aegypti y $A$. albopictus principalmente y Ochlerotatus, reconociéndose actualmente al menos 22 especies vectores en distintas regiones biogeográficas ${ }^{1,2}$. El virus tiene cuatro serotipos, DENV-1 a DENV-4, asociados a dos cuadros clínicos fundamentales: el dengue clásico principalmente asociado a DENV-1 y el dengue hemorrágico asociado a los otros serotipos $^{3-5}$. Aunque el dengue clásico es un cuadro más bien benigno, el dengue hemorrágico puede llegar a mortalidades que fluctúan entre 1 y $5 \%{ }^{6}$.

El dengue es la enfermedad transmitida por artrópodos de mayor morbi-mortalidad en el mundo ${ }^{5}$ y una de las más frecuentes causas de hospitalización en zonas endémicas constituyéndose en la décima causa de muerte por infección en el mundo ${ }^{7}$. Afecta a países tropicales y subtropicales de Asia, Islas del Pacífico, Islas del Caribe, México, África y Centro y Sudamérica ${ }^{1}$. Predomina levemente en mujeres y las edades más afectadas en las zonas endémicas son 13 a 14 años ${ }^{1}$. Se calcula en 2.500 millones la población en riesgo, con 50 a 100 millones de casos al año de fiebre por dengue y 250.000 a 500.000 casos de dengue hemorrágico con alrededor de 30.000 muertes $^{7}$. Hoy es considerada una enfermedad infecciosa emergente y un problema global de salud ${ }^{1}$ que ha incrementado significativamente su rango de distribución y el número de $\operatorname{casos}^{3-5}$, especialmente en América en los países: Brasil, Colombia, Cuba, Ecuador, Perú, Paraguay, Venezuela y Bolivia ${ }^{5}$, lo que se ha atribuido al aumento poblacional, la urbanización no planificada, inadecuado suministro de agua, dificultades en la recolección de residuos sólidos y al incremento en los viajes ${ }^{3}$. Esto se suma a las dificultades en el manejo de las poblaciones de Aedes sp. que desarrollan sus estados inmaduros en pequeños cuerpos de agua, envases, latas, barriles, neumáticos, floreros etc ${ }^{2}$.

En Chile continental hubo poblaciones de A. aegypti cuyos últimos reportes datan de $1961^{8}$. Hasta 1953 , en Chile continental la infestación domiciliaria iba desde el nivel del mar hasta $1.320 \mathrm{msnm}$ en el oasis de Pica $\left(69^{\circ} 18^{\prime} \mathrm{O} / 20^{\circ} 28^{\prime} \mathrm{S}\right)$ y desde Arica a Caldera $\left(71^{\circ} 00 \mathrm{O} / 27^{\circ} 15^{\circ} \mathrm{S}\right)$ alcanzando un máximo de $45,2 \%$ de infestación domiciliaria en Taltal ${ }^{9,10}$.

Hay reportes de casos de dengue (aparentemente dengue clásico) en Iquique en $1889^{8}$. Hasta 1999 no se habían detectado posibilidades de reaparición del vector 
y la enfermedad. Sin embargo, inesperadamente, ya que la principal preocupación era el norte de Chile, en el año 2000 se detectaron poblaciones de A. aegypti en Isla de Pascua $^{11}$. El 13 de marzo de 2002 se confirmó el primer caso autóctono de dengue que podría haberse originado a partir de viajeros infectados, ya que en Tahití y Hawai circuló el virus en el año 200111,12. En 15 semanas se notificaron 636 casos confirmados ( $17 \%$ de la población) produciendo el cuadro clásico y correspondiendo al serotipo DENV-1 en $100 \%$ de los casos ${ }^{12}$. Posteriormente, ha reaparecido en 2006-2007 y 2008, 2009 y 2011 con 3, 27, 25, 25 y 1 casos, respectivamente.

Los modelos matemáticos y los conceptos derivados de ellos, como el número reproductivo, han demostrado gran utilidad para el manejo de enfermedades infecciosas, especialmente en situaciones epidémicas ${ }^{13-20}$. Se han usado tanto en enfermedades de transmisión directa como indirecta y en enfermedades transmitidas por vectores ${ }^{18}$. En Chile se han usado en enfermedades infecciosas de transmisión respiratoria y digestiva e incluso en la enfermedad de Chagas, también transmitida por un vector ${ }^{21-35}$. Todos los modelos determinísticos para transmisión de enfermedades infecciosas mediante vectores biológicos derivan del modelo básico de Macdonald-Ross para la transmisión de la malaria ${ }^{18,36}$, incluidos los modelos para dengue desarrollados por Bailey ${ }^{18} \mathrm{y} \mathrm{Dietz}^{37}$. Aunque existen modelos estocásticos ${ }^{18}$ y diversos modelos más sofisticados ${ }^{38-41}$, la dinámica del dengue se encuentra bien representada en el modelo compartimental más simple que considera susceptibles, infectados y recuperados en la población humana y vectores susceptibles e infectados ${ }^{36}$.

Si los modelos matemáticos representan adecuadamente la dinámica del dengue, se puede esperar que la epidemia de Isla de Pascua en 2002 sea adecuadamente descrita por ellos y que los casos y eventos posteriores sean predichos, contribuyendo a un adecuado manejo de la situación epidemiológica. En este trabajo, nuestro objetivo fue estimar los parámetros epidemiológicos más relevantes de la dinámica de transmisión del dengue en Isla de Pascua y modelar la dinámica desde 2002, comparando las predicciones con la situación real observada.

\section{Material y Métodos}

Fuente de información: Fue el registro de los casos de notificación obligatoria desde 2002 aportados por el Ministerio de Salud.

Estadística básica: Se describe el número de casos, su distribución por edades, edad promedio de infección $(E)$, distribución por sexos y distribución temporal. Se realizó estadística descriptiva con histogramas de frecuencias, promedios y desviación estándar.
Parámetros epidemiológicos: El parámetro epidemiológico fundamental de la trasmisión de enfermedades infecciosas es el número reproductivo $(R o)$ que se define como el número de nuevas infecciones a partir de un caso índice propagadas en un período serial, entendiendo este último como el tiempo que media entre una infección y otra ${ }^{17}$. Hay tres métodos fundamentales para su cálculo:

El método de la edad media de infección: $R_{0}=1+\frac{e_{0}}{E}$ (Eq. 1) donde $e_{0}$ es la esperanza de vida al nacer.

El método de los susceptibles libres de infección $(s)$ al final de la epidemia: $R_{0}=\frac{\ln (s)}{s-1}$ (Eq. 2).

El método de la tasa instantánea de incremento al inicio de la epidemia $\left(r_{0}\right)$, basado en que al inicio de una epidemia el incremento de casos es aproximadamente exponencial: $I=I_{0} \cdot e^{r_{0} t}($ Eq. 3$)$ donde $\mathrm{t}$ es el tiempo, I e $\mathrm{I}_{0}$ el número de casos en cada instante e inicial respectivamente.

Los dos primeros métodos son a posteriori calculándose al final de la epidemia. El tercero es muy útil y puede calcularse al inicio de la epidemia, enfoque que se toma en este trabajo. En el caso de enfermedades transmitidas por vectores, la relación entre $\mathrm{R}_{0} \mathrm{y}_{0}$ que ha sido propuesta es: $R_{0}=\left(1+\frac{r_{0}}{\gamma}\right)\left(1+\frac{r_{0}}{\mu_{v}}\right) \cdot e^{r_{0}\left(\tau_{h}+\tau_{v}\right)}($ Eq. 4) donde $\gamma$ es la tasa de recuperación, $\mu_{\mathrm{v}}$ la tasa de mortalidad del vector y $\tau_{h} \mathrm{y} \tau_{v}$ los períodos de incubación intrínseco (en el hombre) y extrínseco (en el vector) respectivamente ${ }^{42}$.

La tasa $r_{0}$ fue estimada mediante análisis de regresión entre $\ln$ (casos totales) y t, $\gamma=1 / 6$ días $^{-1}, \mu_{v}=0,102$ días $^{-1}$, $\tau_{h}=5$ días y $\tau_{v}=10,22$ días $^{1,42}$.

Ya que en poblaciones cerradas se espera que el dengue evolucione en epidemias recurrentes ${ }^{18,36}$, se calculó el período interepidémico $(\tau)$ como: $\tau=2 \pi \sqrt{\left(D+D^{\prime}\right) E}$ (Eq. $5)$, donde $\mathrm{D}$ y $\mathrm{D}^{\prime}$ son el período infeccioso y el período latente promedio, respectivamente.

El modelo y las simulaciones se realizaron con ecuaciones de diferencias (modelo discreto) basados en los modelos básicos de Dietz y Bailey ${ }^{36}$ (Figura 1):

$S_{h(t+1)}=S_{h(t)}+\eta S_{h(t)}+\mu_{h}\left(N_{h(t)}-S_{h(t)}\right)-\frac{\beta_{h} b}{N_{h}+m} S_{h(t)} I_{v(t)}$

$I_{h(t+1)}=I_{h(t)}+\frac{\beta_{h} b}{N_{h}+m} S_{h(t)} I_{v(t)}-\left(\mu_{h}+\gamma\right) I_{h(t)}$

$R_{h(t+1)}=R_{h(t)}+\gamma I_{h(t)}-\mu_{h} R_{h(t)}$

$S_{v(t+1)}=S_{v(t)}+A-\frac{\beta_{v} b}{N_{h}+m} S_{v(t)} I_{h(t)}-\mu_{v} S_{v(t)}$

$I_{v(t+1)}=I_{v(t)}+\frac{\beta_{v} b}{N_{h}+m} S_{v(t)} I_{h(t)}-\mu_{v} I_{v(t)}$

Además se consideraron las relaciones básicas:

$N_{h(t)}=S_{h(t)}+I_{h(t)}+R_{h(t)}$

$N_{v(t)}=S_{v(t)}+I_{v(t)}$

$N_{v(t+1)}=N_{v(t)}+A-\mu_{v} N_{v(t)}$

(Eqs. 11-13) 
Figura 1. Modelo de transmisión del virus dengue. Los parámetros se encuentran definidos en el texto.
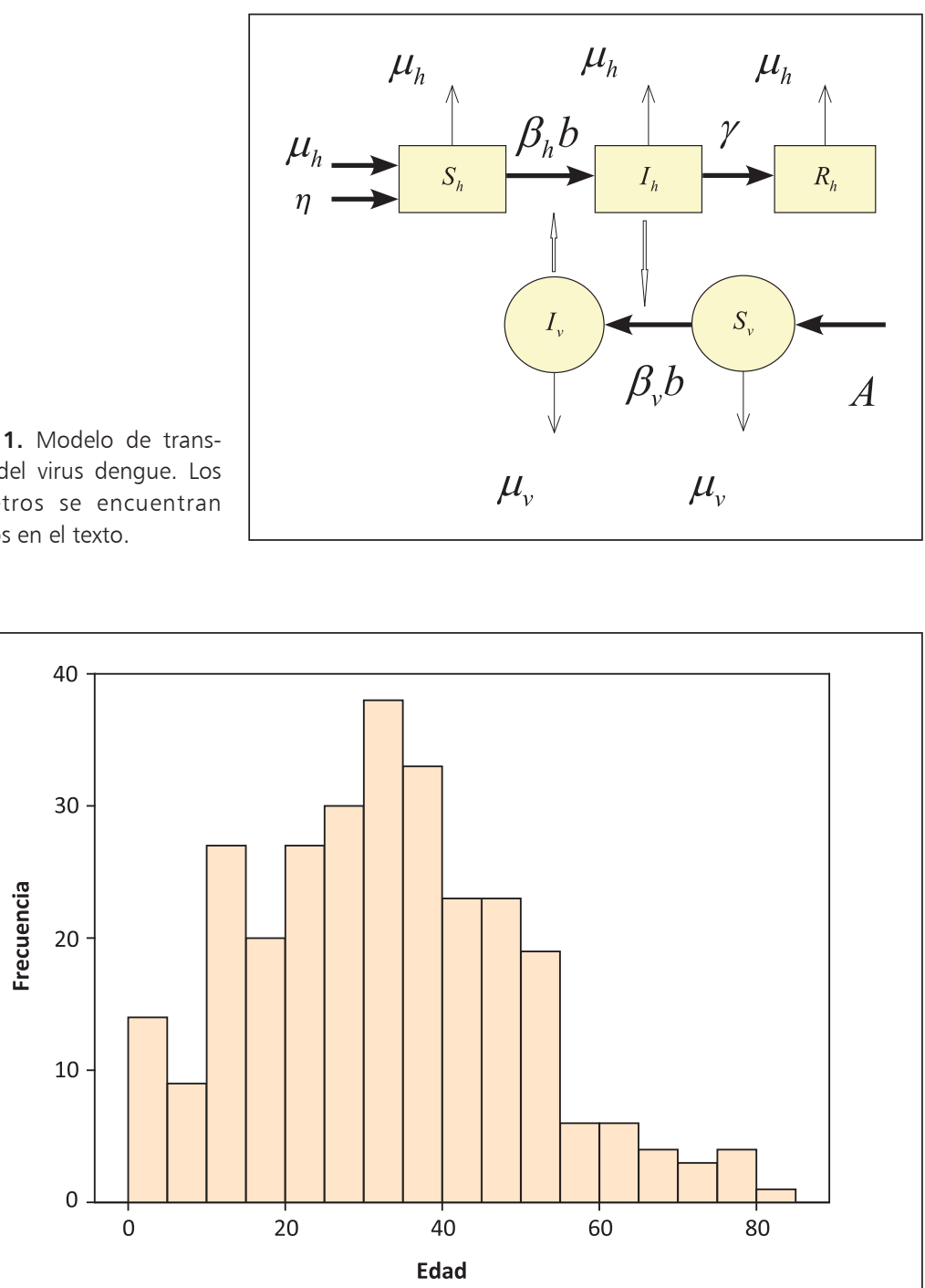

Figura 2. Distribución de frecuencias de los grupos etarios afectados por dengue en Isla de Pascua en 2002.
En estas ecuaciones $S, I, R$ y $N$ representan las poblaciones de susceptibles, infectados, recuperados y total para la población humana (subíndice $h$ ) y del mosquito (subíndice $v$ ) en tiempos sucesivos (subíndices $(t)$ y $(t+1)$. Además $h$ es la tasa de inmigración de la población humana, $\mu$ la tasa de mortalidad de humanos y mosquitos (subíndices $h$ y $v$ ), $\beta$ las probabilidades de transmisión desde el vector al humano (subíndice $h$ ) y desde el humano al vector (subíndice v), $b$ la tasa de picada del mosquito (i.e. número de picadas por mosquito por día), $\mathrm{m}$ el número de hospederos alternativos, $A$, la tasa de reclutamiento del vector. Los valores de los parámetros fueron obtenidos de la literatura científica o calculados (Tabla 1).

La tasa de mortalidad humana en Isla de Pascua fue estimada como $1 / e_{0} \mathrm{y}$ la tasa de reclutamiento del vector
Tabla 1. Valores estimados de los parámetros usados en

el modelo

\begin{tabular}{|ccc|}
\hline Parámetro & Valor & Fuente \\
$\boldsymbol{\mu}_{\boldsymbol{h}}$ & 0,0000383 días $^{-1}$ & Estimado \\
$\boldsymbol{\mu}_{\boldsymbol{v}}$ & 0,102 días $^{-1}$ & 42 \\
$\boldsymbol{\beta}_{\boldsymbol{h}}$ & 0,4 & 36 \\
$\boldsymbol{\beta}_{\boldsymbol{v}}$ & 0,4 & 36 \\
$\boldsymbol{B}$ & 0,7 picadas/díaxinsecto & 28 \\
$\boldsymbol{M}$ & 0 & 36 \\
$\boldsymbol{\gamma}$ & 0,167 días $^{-1}$ & 1 \\
$\boldsymbol{A}$ & 802 insectos $^{-1}$ & Estimado \\
$\boldsymbol{e}_{\boldsymbol{\theta}}$ & 71,6 años & Censo 2002 \\
\hline
\end{tabular}

fue obtenida de la relación:

$$
R_{0}=\sqrt{\frac{b^{2} \beta_{h} \beta_{v} N_{h} A / \mu_{v}}{\left(N_{h}+m\right)^{2} \mu_{v}\left(\gamma+\mu_{h}\right)}}
$$

(Eq. 14) $)^{36}$ donde $A$ es la incógnita y $R_{0}$ fue estimado a partir de los datos.

\section{Resultados}

De los 636 casos confirmados reportados por el MINSAL, 551 casos se encontraron en los registros consultados. Estos corresponden en 52,27\% a mujeres y 47,73\% a hombres. La edad promedio de infección fue $\mathrm{E}=31,38$ $\pm 18,37$ años, siendo $\mathrm{E}=32,65 \pm 17,06$ años en mujeres y $\mathrm{E}=29,82 \pm 19,76$ años en hombres. La distribución tuvo una pequeña asimetría negativa, con una mediana $\mathrm{Me}=32,58$ años y una moda de 37,25 años (coeficiente de asimetría de Pearson $\gamma_{3}=-0,32$; Figura 2). Mediante la Eq. 1, la edad media de infección permitió una primera aproximación puntual al número reproductivo $\mathrm{R}_{0}=3,28$.

El quiebre en la curva de progresión de casos acumulados se produjo alrededor del día 125. Esto permitió obtener un buen ajuste $\left(\mathrm{F}_{1,5}=19,74 ; \mathrm{p}=0.007 ; \mathrm{R}^{2}=0,7\right)$ entre el logaritmo natural de I y $\mathrm{t}$ (ver Eq. 3 ) para el cálculo de $r_{0}$ (Figura 3). Se obtuvo $r_{0}=0,038 \pm 0,008$ días $^{-1}$ con un intervalo del $95 \%$ de confianza $\mathrm{IC}_{0,95}=[0,022 ; 0,054]$. Basados en la Eq. 4, se estimó un número reproductivo $R_{0}=3,005$ con un $\mathrm{IC}_{0,95}=[1,92 ; 4,61]$.

Considerando que el período de latencia típicamente $\mathrm{D}$ es entre 3 y 7 días y que el período infeccioso $D^{\prime}$ es entre 5 y 7 días $^{1}$ el período inter-epidémico esperado puede ir entre $\tau=5,20$ y 6,8 años (Eq. 5). Por ejemplo, para el serotipo DENV-1, se ha reportado un período de incubación de 5,7 $\pm 1,5$ días y un período infeccioso de 5 días $^{38}$. Además reportan posibilidad de contagio dos días antes del inicio de los síntomas, lo que implica un período de latencia aproximado de 3,7 días, lo que implicaría un $\tau=5,42$ años. 
Basados en $R_{0}=3,005$, la proporción de individuos infectados al final de la epidemia (Eq. 6) resultó $p=1-s$ $=94 \%$ con un $\mathrm{IC}_{0,95}=[78,0 ; 98,5]$.

La simulación de casos muestra una dinámica caracterizada por epidemias recurrentes que van decreciendo en magnitud (oscilaciones amortiguadas), lo que es un hecho conocido en todos los modelos de dengue y malaria ${ }^{18,36}$, y presenta un buen ajuste cualitativo a la dinámica epidemiológica desde 2002 en adelante. Predice adecuadamente el alza de casos entre 2006 y 2011. El número de casos predichos durante la epidemia de 2002 es mayor que los casos confirmados y el curso de la epidemia predicha es más acelerado. El período inter-epidémico en la simulación es de 6,72 años entre 2002 y 2008 y 4,68 años entre 2008 y 2013 (Figura 4). Desde la perspectiva teórica la primera epidemia debió afectar a $94 \%$ de la población (aproximadamente 3.500 casos); sin embargo, se notificaron 639. Para el segundo brote con pico en 2008 se esperaban 930 casos y para 2013 se esperarían 759 casos.

Sin considerar cambios en las tasas de migración mortalidad ni natalidad, la simulación a largo plazo muestra epidemias recurrentes de dengue (DENV-1) decrecientes en magnitud hasta su extinción (Figuras 5 y 6 ) en aproximadamente 20 años a contar de 2002.



Figura 3. Evolución en el tiempo de los casos acumulados y confirmados de dengue en Isla de Pascua en 2002.

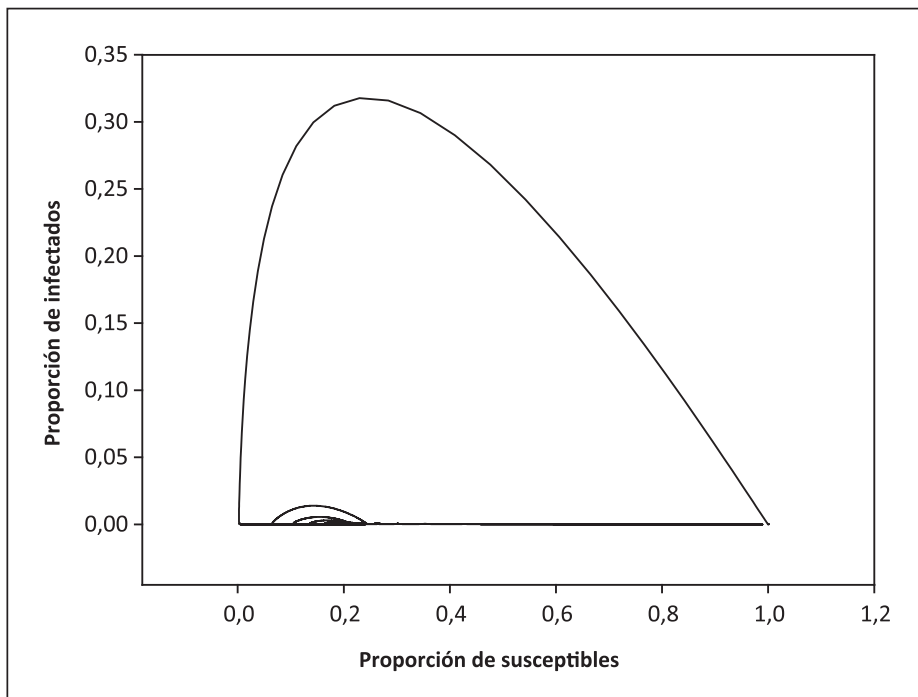

Figura 5. Fluctuaciones de las proporciones de infectados y susceptibles en una simulación a largo plazo.

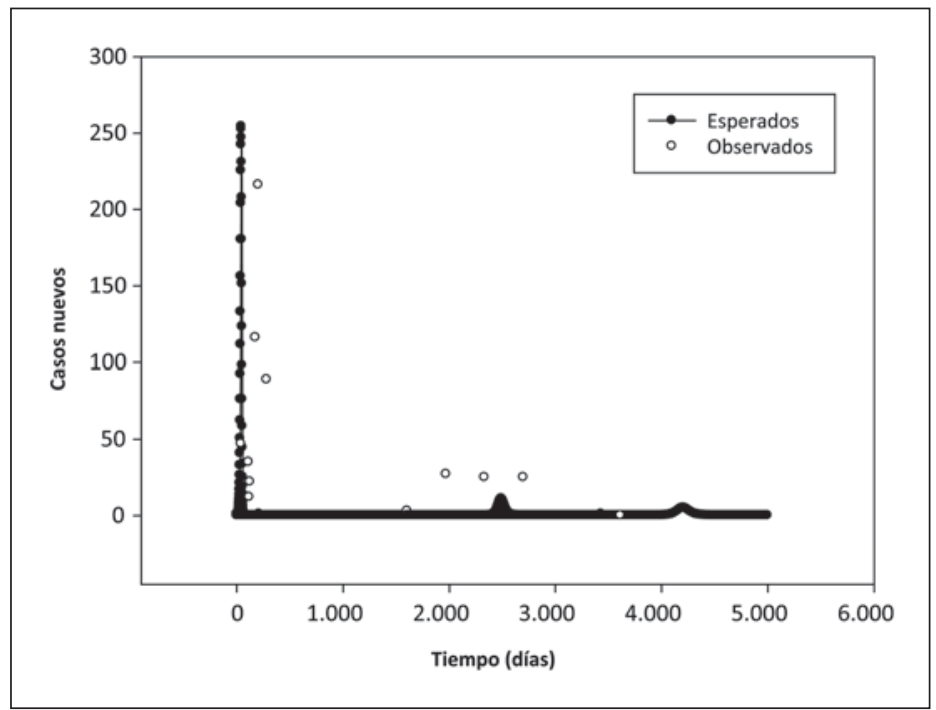

Figura 4. Comparación entre los casos confirmados y los casos esperados mediante simulación con ecuaciones de diferencia para el dengue en Isla de Pascua en 2002.

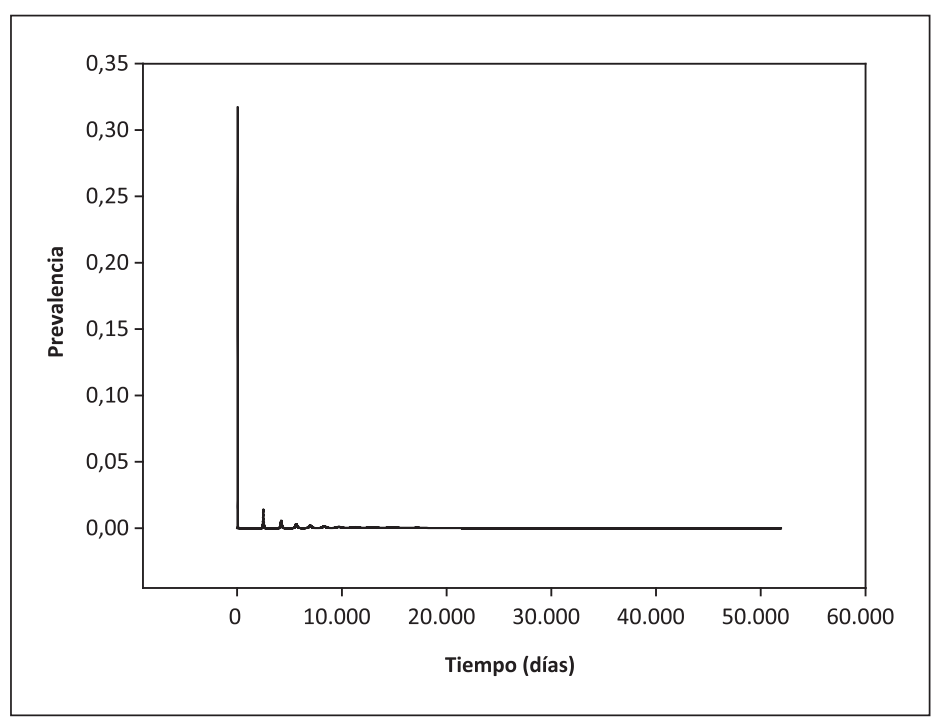

Figura 6. Variación en la incidencia de casos a lo largo del tiempo en una simulación a largo plazo. 


\section{Discusión}

La epidemia de dengue-2002 en Isla de Pascua sorprendió a una población con $100 \%$ de susceptibles. La edad media de infección cercana a los 30 años es mayor que la reportada en países donde el dengue es endémico en los cuales el grupo de mayor riesgo está entre los 13 y 16 años ${ }^{1}$, lo que es explicado por el primer enfrentamiento de una población susceptibles al virus (DENV-1). En posteriores epidemias se podría esperar un descenso en la edad media de infección y en el período inter-epidémico, ya que los susceptibles se recuperan en base a los nacimientos, reclutando casos a edades menores. Una excepción a esto sería el caso de un aumento poblacional por inmigración, lo que implicaría un ingreso de susceptibles de edades mayores. La primera estimación al número reproductivo $R_{0}=3,28$ resultó bastante consistente con la estimación basada en el reclutamiento de casos $R_{0}=3,01$ a pesar de las debilidades que tiene el basarse en los casos notificados y confirmados. Estos últimos pierden la temporalidad al ser reportados cada semana y entonces no es posible determinar la exacta prevalencia. Por este motivo el cálculo se basó en los casos acumulados que al inicio de las epidemias siguen un comportamiento casi exponencial (la curva logística y la exponencial casi se sobreponen).

Basados en nuestros cálculos se puede sugerir que al inicio de la epidemia cada caso índice producía alrededor de tres casos secundarios. Aunque existen muy variadas estimaciones de $R_{0}$ para el dengue, nuestro valor es consistente con los trabajos más actuales en la materia. Por ejemplo, mientras las primeras estimaciones sugerían un $R_{0}=27^{37}$, las estimaciones posteriores son más consistentes con valores dentro de nuestro intervalo de confianza $[1,92 ; 4,61]$. Por ejemplo se ha reportado $R_{0}=1,3$ en México $1991^{43}, R_{0}=1,9^{44}, R_{0}=1,6-2,5^{45}$ en Brasil, $R_{0}=4,3-5,8$ en Tailandia ${ }^{46}, R_{0}=2,7-11,6$ en Brasil $^{47,48}$ y $R_{0}=3,8-5,1^{42}$. El valor de $R_{0}=3,01$ permite estimar el número potencial de picadas infectantes que puede distribuir la población de mosquitos $A$. aegypti a partir de un caso índice, lo que corresponde al concepto de capacidad vectorial ${ }^{49-51}\left(C V=R_{0} \gamma\right)^{52}$. Este valor es $C V=0,502$, lo que resulta consistente con estimaciones obtenidas para este mosquito en Río de Janeiro para un valor semejante de sobrevivencia $\left(p=e^{-\mu}\right): C V=0,4215^{53}$. La eficiencia vectorial ${ }^{51}$, entendida como la probabilidad de producir infección cuando el mosquito porta el virus (producto de la probabilidad de transporte por la probabilidad de transmisión), en nuestras simulaciones llegó a un máximo de 0,165 en el pico de la epidemia.

La proporción esperada de infectados al final de la epidemia de 2002, basada en $R_{0}=3,005$ fue de $94 \%$, lo que sugiere que habrían ocurrido aproximadamente 3.500 casos. Sin embargo, fueron declarados sólo 636 . De ser esto correcto, indicaría que de los casos de dengue clásico (DENV-1) sólo 18,2\% llega a declararse. Esto implicaría gran cantidad de casos leves o subclínicos. En el Ministerio de Salud se ha propuesto que la epidemia habría afectado a $80 \%$ de los susceptibles ${ }^{12}$, lo que es algo menor que nuestros cálculos pero dentro del intervalo de confianza (aproximadamente 3.000 casos reales). Sin embargo, coincide con la proposición de gran cantidad de casos subclínicos.

La dinámica predicha se caracteriza por epidemias recurrentes de magnitud decreciente. El período interepidémico calculado varía entre 5,2 y 6,8 años, lo que es aproximadamente coincidente con lo ocurrido entre 2006 y 2009, período en que se reportaron 80 casos $^{12}$. La simulación sugiere una primera epidemia con 3.500 casos ( $94 \%$ de los susceptibles), una segunda epidemia de 930 casos con pico en 2008 y se esperaría un tercer brote con pico en el año 2013, de 759 casos. Si se mantiene la baja proporción de notificación (18\%), la segunda epidemia debería haber tenido aproximadamente 167 casos notificados (vs 80) y en la siguiente haber alrededor de 140 casos. Las simulaciones son consistentes con lo que ha ocurrido desde 2002 y consistentes con la dinámica del dengue en diversas partes del mundo ${ }^{18}$. A largo plazo se esperarían epidemias recurrentes de menor magnitud que condujeran a una extinción del dengue en alrededor de 20 años. Sin embargo, esta predicción es sólo válida para DENV-1, ya que la inmunidad adquirida no protege contra los otros serotipos. Esto produce un serio problema, ya que si la población de $A$. aegypti se mantiene en Isla de Pascua, la posibilidad de una epidemia de dengue hemorrágico es altísima y sólo depende de la posible introducción de los otros serotipos desde la polinesia u otras regiones del globo. Ya existe cierta preocupación por la existencia de tres casos por DENV-4 en 2009. En Cuba en 1981 ocurrió una gran epidemia de dengue hemorrágico (por DENV-2) de miles de casos, a continuación de la epidemia de dengue clásico (por DENV-1) en 1977-78 54 un rebrote en $1997^{55}$.

Agradecimientos. Los autores agradecen a Maritza García. Jefa de la Unidad de Enfermedades Transmisibles del Ministerio de Salud, a Andrea Olea y Fernando Fuenzalida del Departamento de Epidemiología del Ministerio de Salud, quienes facilitaron los datos que forman parte de este estudio y a Carlos Pavletic y Alonso Parra del Programa Nacional de Control de Vectores, Departamento de Salud Ambiental, División de Políticas Públicas Saludables y Promoción del Ministerio de Salud.

\section{Resumen}

El dengue es considerado una enfermedad emergente que aumenta su prevalencia especialmente en Sudamérica. En 2002 ocurrió inesperadamente una epidemia de dengue clásico (DENV-1) en Isla de Pascua, hecho nunca antes 
detectado. Posteriormente, ha reaparecido en 2006-2007 y 2008, 2009 y 2011. El objetivo de este trabajo fue estimar los parámetros epidemiológicos más relevantes de la dinámica de transmisión del dengue en Isla de Pascua y modelar la dinámica desde 2002, comparando las predicciones con la situación real observada. Del total de casos, 52,27\% correspondieron a mujeres y $47,73 \%$ a hombres. La edad promedio de infección fue $E=31,38$ $\pm 18,37$ años, similar en hombres y mujeres. Se estimó el número reproductivo $R_{0}=3,005$ con un $\mathrm{IC}_{0,95}=[1,92$; $4,61]$. El período inter-epidémico esperado puede ir entre $T=5,20$ y 6,8 años. La simulación de casos mostró una dinámica con epidemias recurrentes que van decreciendo en magnitud (oscilaciones amortiguadas), lo que es un hecho conocido en todos los modelos de dengue y malaria.
Hubo un buen ajuste cualitativo a la dinámica epidemiológica desde 2002 en adelante. Se predijo adecuadamente el alza de casos entre 2006 y 2011. El número de casos predichos durante la epidemia de 2002 es mayor que los casos confirmados y el curso de la epidemia predicha es más acelerado. El período inter-epidémico en la simulación es de 6,72 años entre 2002 y 2008 y 4,68 años entre 2008 y 2013. Desde la perspectiva teórica, la primera epidemia debió afectar a 94\% de la población (aproximadamente 3.500 casos); sin embargo, se notificaron 639 lo que sugiere sub-notificación y gran cantidad de casos sub-clínicos. Se esperan futuros rebrotes epidémicos de tamaño decreciente, aunque se advierte que el principal peligro radica en epidemias de dengue hemorrágico, producto de la introducción de los otros serotipos.

\section{Referencias bibliográficas}

1.- Acha P, Szifres B. Zoonosis y enfermedades transmisibles comunes al hombre y a los animales. Vol II. Dengue. Publicación científica y técnica OPS 2003; 580: 66-71.

2.- González GR, Mac Lean M. Moscas y mosquitos. (En Canals M \& PE Cattan (2008) Zoología médica II. Invertebrados. Santiago, Editorial Universitaria. Pp: 299-353.

3.- Valero N, Reyes I, Larreal Y, Maldonado M. Aminotransferasas séricas en pacientes con Dengue tipo 3. Rev Med Chile 2007; 135: 1304-12

4.- Rojas E, Díaz-Quijano FA, Coronel-Ruiz C, Martínez-Vega RA, Rueda E, Villar-Centeno L A. Correlación entre los niveles de glutatión peroxidasa, un marcador de estrés oxidativo, y la presentación clínica del dengue. Rev Med Chile 2007; 135: 743-50.

5.- Martínez-Vega R A, Díaz-Quijano F A, Villar-Centeno L A. Dificultad para el diagnóstico clínico temprano del dengue en un área endémica y su impacto sobre el manejo médico inicial. Rev Med Chile 2006; 134: 1153-60.

6.- MINSAL en http://epi.minsal.cl/epi/html/enfer/ PrevDengueWeb.pdf. (accedido en noviembre de 2011).

7.- Seijo A. El dengue como problema de salud pública. Arch Argent Pediatr [online] 2001; 99 (6). En:http://www.sap.org.ar/staticfiles/ archivos/2001/arch01_6/510.pdf. (accedido en noviembre de 2011).

8.- Laval E. ¿Hubo dengue autóctono en Chile? Rev Chilena Infectol. Edición aniversario 2003; 98-9.

9.- Neghme A. Control del A. aegypti en Chile. Bol Of Sanit Panam 1950; Abril: 389.

10.- Neghme A, Albi H, Gutiérrez J. Campaña de erradicación del Aedes aegypti en Chile. Bol Of Sanit Panam 1953; 34 (3): 205-20.

11.- Olea P. Primer caso de dengue autóctono atendido en el Hospital de Enfermedades
Infecciosas Dr. Lucio Córdova. Rev Chilena Infectol 2003; 20 (2): 129-32.

12.- MINSAL. Dengue en Isla de Pascua. Situación Epidemiológica al 14 de Octubre de 2011. http://epi.minsal.cl/epi/html/bolets/reportes/ Dengue/Informedengue.pdf. (accedido en noviembre de 2011).

13.- Anderson R. Epidemiology. In: Cox FEG (ed) Modern Parasitology: 75-116. Blackwell Scientific Publications, Oxford. 1993.

14.- Anderson R, May R. Population biology of infectious diseases: Part I. Nature 1979; 280: 361-7.

15.- Anderson R, May R. Population biology of infectious diseases. Report of the Dahlem Workshop on population biology of infectious disease agents. (RM Anderson \& RM May Eds). 1982; Sciences report 25. Dahlem Konferenzen. Springer Verlag. Berlin and New York.

16.- Anderson R, May R. Infectious diseases of humans: Dynamic and control. 1991; Oxford University Press, Oxford.

17.- Heffernan JM, Smith RJ, Wahl LM. Perspectives on the basic reproductive ratio. J R Soc Interface 2005; doi: 10.1098/ rsif.2005.0042: 1-13

18.- Bailey N. The mathematical theory of infectious diseases and its applications. 1975; Griffin, London.

19.- May R. Ecological aspects of diseases and human populations. Am Zool 1985; 25 : 441-50.

20.- May R, Anderson R. Population biology of infectious diseases: Part II. Nature 1979; 280 : 455-61.

21.- Canals M. Sarampión en Chile: una peligrosa onda. Rev Med Chile 1992; 120: 585-8.

22.- Canals M. Dinámica de transmisión de enfermedades infecciosas: una expresión particular de una dinámica depredador-presa. Rev Med Chile 1993; 121: 1316-20.

23.- Canals M, Cattan P E. Dinámica de transmisión de la infección chagásica en Chile: Modelo y simulación. Rev Med Chile 1992; 120 : 1359-65.

24.- Canals M, Labra F. Análisis no-lineal de la dinámica de enfermedades infecciosas en Chile. Rev Med Chile 1999; 127: 1086-92.

25.- Canals M, Martínez L, Firinguetti L. Dinámica ecológica de enfermedades infecciosas: Parte I Estacionalidad. Rev Med Chile 1989; 117: 87-94.

26.- Canals M, Martínez L, Firinguetti L. Dinámica ecológica de enfermedades infecciosas: Parte II Recurrencia epidémica periódica. Rev Med Chile 1989; 117: 95-100.

27.- Canals M. Dinámica epidemiológica de la escarlatina en Chile. Rev Chilena Pediatr 1989; 60 (1): $15-8$.

28.- Canyon DV, Hii J L K, Muller R. The frequency of host biting and its effect on oviposition and survival in Aedes aegypti (Diptera: Culicidae). Bull Ent Res 1999; 89: 35-9.

29.- Canals M. Una aproximación a la dinámica de la meningitis meningocócica mediante sistemas dinámicos y series de tiempo. Rev Med Chile 1996; 124:178-88.

30.- Scortti M, Cattan P E, Canals M. Proyecciones de rabia canina en Argentina, Bolivia y Paraguay, usando series de tiempo. Arch Med Vet 1997; 29 (1): 83-9.

31.- Canals M, Labra F. Meningitis y Caos. Rev Med Chile 1997; 125: 1110-1.

32.- Canals M. Inicio de la pandemia AH1N1: Algebra, cálculo y geometría del contagio. Rev Med Chile 2009; 137: 852-8.

33.- Canals M. Predictibilidad a corto plazo del número de casos de la influenza pandémica AH1N1 basada en modelos determinísticos. Rev Chilena Infectol 2010; 27 (2): 114-20.

34.- Canals M, Canals A. Percolación de la epidemia A (H1N1) en el mundo: utilidad de los modelos predictivos basados en conectividad espacial. Rev Med Chile 2010; 138: 573-80.

35.- Canals M. Análisis comparado de la dinámica 
epidemiológica de la influenza A(H1N1) en Chile. Rev Med Chile 2010; 138: 1179-89.

36.- Nishiura H. Mathematical and statistical analyses of the spread of dengue. Dengue Bull 2006; 30: 51-67.

37.- Dietz K. Transmission and control of arbovirus diseases. In: Ludwig D, Cooke KL editors. Epidemiology. SIAM, Philadelphia, 1975. p. 104-21.

38.- Nishiura H, Halstead SB. Natural history of dengue virus (DENV)-1 and DENV-4 infections: reanalysis of classic studies. J Infect Dis 2007; 195: 1007-13.

39.- Basáñez M G, Rodríguez D J. Dinámica de transmisión y modelos matemáticos en enfermedades transmitidas por vectores Entomotropica 2004; 19 (3): 113-34.

40.- Pongsumpun P. Transmission model for dengue disease with and without the effect of extrinsic incubation period KMITL Sci Tech J 2006; 6 (2): 74-82.

41.- Pongsumpun P. Mathematical model of dengue disease with the incubation period of virus. World Academy of Science, Engineering and Technology 2008; 44: 328-32

42.- Favier C, Degallier N, Rosa-Freitas M G, Boulanger J P, Costa Lima J R, LuitgardsMoura J F, et al. Early determination of the reproductive number for vector-borne diseases: the case of dengue in Brazil. Trop Med Int Health 2006; 11 (3): 332-40.

43.- Koopman J S, Prevots D R, Vaca Marín M A, Gomez Dantes H, Zarate Aquino ML, Longini IM Jr, et al. Determinants and predictors of dengue infection in Mexico. Am J Epidemiol 1991; 133 (11): 1168-78.

44.- Newton E A, Reiter P. A model of the transmission of dengue fever with an evaluation of the impact of ultra-low volume (ULV) insecticide applications on dengue epidemics. Am J Trop Med Hyg 1992; 47 (6): 709-20.

45.- Marques C A, Forattini O P, Massad E. The basic reproduction number for dengue fever in Sao Paulo State, Brazil: 1990-1991 epidemic. Trans R Soc Trop Med Hyg 1994; 88 (1): 58-9.

46.- Ferguson N M, Donnelly C A, Anderson R M. Transmission dynamics and epidemiology of dengue: insights from age-stratified seroprevalence surveys. Philos Trans R Soc Lond B Biol Sci 1999; 354 (1384): 757-68.

47.- Massad E, Coutinho FA, Burattini MN, López LF. The risk of yellow fever in a dengue-infested area. Trans R Soc Trop Med Hyg 2001; 95 (4): 370-4.

48.- Massad E, Burattini M N, Coutinho F A, López L F. Dengue and the risk of urban yellow fever reintroduction in Sao Paulo State, Brazil. Rev Salud Pública 2003; 37(4): 477-84.
49.- Garret-Jones. Malaria vectorial capacity of a population of Anopheles gambiae. An exercise in epidemiological entomology. WHO Bull 1969; 40: 531-45.

50.- Lehane M J. Biology of blood sucking insects. 1991. Harper Collins Academics, London.

51.- Canals M, Bustamante R O, Ehrenfeld M, Cattan P E. Assessing the impact of insect vectors on animal populations. Acta Biotheor 1999; 46: 337-45.

52.- Wernsdofer W H, McGregor I. Malaria: Principles and Practice of Malariology. Edinburgh: Churchill Livingstone; 1989.

53.- Maciel de Freitas R, Eiras A E, Lourenco de Oliveira R. Calculating the survival rate and estimated population density of gravid Aedes aegypti (Diptera, Culicidae) in Rio de Janeiro, Brazil. Cad Saúde Pública, Rio de Janeiro 2008; 24 (12): 2747-54

54.- Dotres C, Fallat G, Martínez E, Carpio R, Hernández E, Rojo M. Algunos aspectos clínicos durante la epidemia del dengue en Cuba. Cad Saúde Pública 1987; 2 (3): 148-57.

55.- Valdés L, Guzmán M G, Kourí G, Delgado J, Carbonell I, Cabrera M V, Rosario D, Vázquez S. La epidemiología del dengue y del dengue hemorrágico en Santiago de Cuba, 1997. Rev Panam Salud Pública 1999; 6 (1): $16-25$. 